\title{
Dynamic Phase Changes: Integrating Information in Complex Biological Systems
}

\author{
Trevor N. Carniello \\ Laurentian University, Sudbury, Ontario, Canada P3E 2C6 \\ tn_carniello@laurentian.ca
}

\begin{abstract}
Here we examine the potential relationship between applied exogenous EMFs and their ability to generate phase-modulations with information carrying capacity. We systematically examine, through dimensional analysis, the potential sources and interactions of these generated phase-modulations. Furthermore, we introduce the concept of generating phase-modulated signals through the application of weak, time-varying, amplitude modulated EMFs. Information generated through the magnetic pressure of zero-point fluctuations (i.e. Casimir Effect) are also discussed.
\end{abstract}

\section{Indexing terms/Keywords}

Arahanov-Bohm effect; phase modulation; Casimir effect;

\section{Academic Discipline}

Theoretical physics and information processing

\section{SUBJECT CLASSIFICATION}

Magnetic field interactions; biophysics

\section{TYPE (METHOD/APPROACH)}

Dimensional analysis and quantification

\section{INTRODUCTION}

\section{COMPLEXITY AND ITS RELATION TO SPACE-TIME STRUCTURE}

Complexity describes the behaviour, or series of responses, of a system to a set of stimuli resulting a net alteration in the spatial-temporal composition of the entire set. If the degree of complexity is such that the parameters, which limit the spatial and temporal degrees of freedom of the system of observation, are exceeded then the result can manifest into an emergent property. Here we operationally define complexity as the available degrees of freedom in which information can be transferred (gained or lost) to a system from its environment (surrounding). However, traditionally, complexity is observed to be the degree of ordered states, or structure, which can be acted upon [29,31]. That is to say that complexity, at least from the perspective of space, is static and does not evolve in time. This initial conceptualization of complexity is incomplete as it does not consider the influence of time, or underlying change, as a contributory variable to observed phenomena. In our definition we describe the complexity of a system (under observation) as the sum of its processes. Temporal complexity, under our working hypothesis, would then be the available number of potential interactions, or processes, which can impact the structural complexity of fixed space-like degrees of freedom.

The representation of the entire observable set of parameters or degrees of freedom, the Universe, can then be dichotomized into space and time. More specifically, space and time-like degrees of freedom. Space can be described as a physical substrate which behaves, or more aptly can be modeled, in terms of particulate interactions [50]. Matter is the organization of a discrete set of tangible units into an ordered state. The occupation of space, or the change/distortion of curvature of a subset of space-time proportional to the mass of material [24, 37], would constitute the presence of matter. Due to its spatial properties, matter can be conceptualized as crystalized or fixed whereas energy, the antithesis of matter, would be fluid. The former case, that which is described as having permanence, would allow for the representation of information or energy over protracted periods of time. In contrast, the fluidity of energy does not favour permanence, but the rapid dissipation of organized states. Information exchanged between two fragile albeit ordered states (energy) could only be represented transiently. An exchange between static and fluid (or static and static) states would allow for a prolonged representation of said information. That is to say, that the representation of information (e.g., memory) would be predicated on the presence of a fixed structure.

Time, in our case, evolves and is manifested when matter undergoes a measurable change. The complementary analogy in this context would be that time is reflective of energy or the measured unit (quantum) necessary to elicit a change in the aggregate occupying space. In essence then, the superposition between space and time, which is manifested as space-time, is the interaction or exchange of energy to matter or matter to energy. The whole of the exchange is what we define as a process. If a process is the result of matter interacting with energy, then there should be an identifiable change that occurs with respect to spatial degrees of freedom. Consequently, if energy can be affected by matter then we would expect, in light of our hypothesis, that energy, the representation of the temporal structure of the system, should also undergo a change in its temporal degrees of freedom (i.e. pattern or frequency modulation). How do 
we manipulate both the temporal and spatial parameters of a matter-energy interaction in order to extract useable information?

De Broglie postulated that matter exhibits wave-like properties, an idea which forms one of the cornerstones of quantum mechanics [15]. In addition, energy can be described as a wave-function in quantum mechanics, whereby the collapse in the wave-function of a given state produces a physical particle with the same parameters defined in the original wave-equation $[3,6,28,56]$. If matter and energy can be described as wave-functions, then they would be subject to the dynamics of a wave. A candidate for our investigation would be the phase of the matter and energy waves. If the phase of a matter and energy wave are matched they are said to be "in phase" or "phase-locked". If one of the pair is delayed, say by the presence of inter-stimulus delay, and the pair were to be compared, then one would be out of position, either by some distance (wavelength), or angle, with respect to the other. The net result when examining the superposition of the two, would be a new pattern subject to a series of constructive (in-phase) and destructive (out-of-phase) interferences. Looking at the resultant wave of matter-energy interaction, and the minute fluctuations in the degree of separation between adjacent peaks, would be evidence for phase modulation within the daughter wave [14,48].

\section{PHASE MODULATION AND INFORMATION CARRYING CAPACITY}

Cahn [9] postulated that information can be stored within a phase modulated pattern and could be extracted. In this manner, phase modulation could be representative of the relative exchange of information between two entities or systems. Furthermore, it was reported that that the amount of information carried within a phase-modulated pulse pattern, provided that the number of pulses and the number of phase modulations are equivalent, could be approximated as:

$$
\mathrm{I}=\mathrm{m} \log _{2} \mathrm{~m}
$$

Where $m$ is the number of phase modulations when a multiphase modulation is used [9].

While examining the elegant case of an electron orbiting a proton as modeled by a Bohr atom, we can approximate the number of spatial degrees of freedom, or degree of available spatial phase changes, as being akin to the number of potential locations that a classical electron could occupy within the context of that volume. Provided that the classical radius of an electron is $2.818 \cdot 10^{-15} \mathrm{~m}$ and that the radius of the Bohr atom is approximately $5.29 \cdot 10^{-11} \mathrm{~m}$, the quotient of the volumetric equivalent of the latter and former produces a value of $10^{12}-10^{13}$ possible positions which can be occupied at any given time. Now, in order for the electron to occupy any one given position (degree of freedom), energy must be absorbed or emitted. For instance, if the electron is in its wave state, then energy must be absorbed in order to constrict its size from that of its Compton wavelength to that of its classical wavelength. In this light, Persinger [40] calculated the relative energy necessary to transform the wave-like electron to its matter-like counterpart; the value approached $10^{-20} \mathrm{~J}$. Furthermore, the exchange of a photon between the proton and the electron, provided that the energy is within the appropriate band, can also elicit this form of transition. The exchange of energy from wave to particle, or vice versa, alters the phase of the particle which has been intimately linked to the functional collapse of the wave-function.

Irrespective of the amount of energy involved in the translation of one position, or the functional energy equivalent of the collapse of the Compton wavelength to its classical radius of the electron, the number of discrete phases stay the same. Employing Cahn's equation for the binary information stored within the phase modulation within a signal, yields an approximate amount of information in the order of $3.98 \cdot 10^{13}$ bits of information. This value inherently reflects the amount of information available within a Bohr atom, as well as the potential limit of information processing by the system.

Our provided example dealt with the idea that spatial degrees of freedom correspond to a degree of space-like phase modulations. Classically, however, phase modulation is more generally described as a change in the distance between successive peaks or troughs, of a given wave, and is most widely employed as a means of optical or fiber optic communication and information transfer $[2,5]$. This then directly implicates the photon as the primary mode by which to carrier the information contained in a series of phase modulations. Examining this relationship, Mérolla et al., [34] experimentally demonstrated the ability to encode, send, and receive information using phase modulated light transmission with an emphasis on the practical application of cryptology. Additionally, Sun et al., [55] presented the idea that information could be represented in within the difference between successive wavelengths of light. This suggests that not only is the wavelength of the light carrier important to the information contained within that carrier, but so are the spatial distances between each individual wavelength. Alternatively stated, the breaks or pauses in the carrier wave also contains relevant information.

The photon is conceived to be the carrier of the electromagnetic force and we have presented data to suggest that the photon, and by extension electromagnetic radiation, plays an intimate role in the transmittance of information. On this line, Ikonen and colleagues [29] demonstrated the potential of using an AC magnetic field capable of producing transient phenomena resembling mechanical phase-modulation of ${ }^{67} \mathrm{Zn}$ molecules in $\mathrm{ZnO}$ crystals. This suggests that, if the photon is the carrier particle for the electromagnetic force, then it could be acted on by electromagnetic radiation falling outside the spectrum of "light" ranging from infra-red to ultraviolet. Information transmitted as phase-shifts are not solely limited to photons and can be acted upon by an exogenous electromagnetic field. Fang et al., [26] postulated that the phase of a photon carrier can be modulated using a dynamic Arahanov-Bohm effect.

\section{CHANGING PHASE IN AN AMPLITUDE MODULATED TIME-VARYING FIELD}

We describe phase as the offset in angle, distance, or position of a given point traveling in a wave with respect to a reference wave, at a given time. This can be considered as the structure, or location, equivalent of a particle (matter) or wave (energy) as it progresses through time in relative comparison to a reference structure (position) or pattern. The 


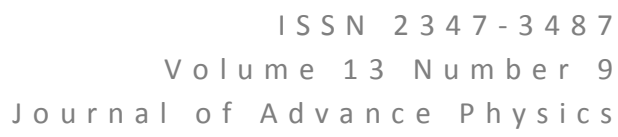

degree of which one can influence the number of phase transitions, would be contingent upon the amount of energy provided to the system. Where a phase transition is the response of a particle's position to the presence of an external modulation or applied process. One way to elicit a phase change in a system is by exposing the particle to an electromagnetic potential and can be modeled using the Arahanov-Bohm [1,57] equation (1).

$$
\Delta \varphi=\frac{q \Phi}{\hbar}(1) .
$$
magnetic flux.

Where, $\Delta \varphi$ is the phase change, $\mathrm{q}$ is the elementary charge, $\hbar$ is reduced Planck's constant and $\Phi$ is the

If the process were occurring in time, then the form would be:

$$
\frac{\Delta \varphi}{t}=\frac{q \Phi}{\hbar t}(2)
$$

Where $t$ is the point duration of a time-varying electromagnetic field.

Now, if the elementary unit charge and Planck's constant do not appreciably change with respect to time (i.e., are constants) then only parameter that is left to change in time is the magnetic flux. Conceptually, magnetic flux is defined as the intensity, the degree of bunching or number of magnetic lines of force, of a magnetic field penetrating a given surface. The resultant mathematical representation would take on the form of:

$$
\Phi=B \cdot A(3)
$$

Where $B$ is the intensity of the magnetic field (Tesla) and the A is the surface area $\left(\mathrm{m}^{2}\right)$ of a material which is immersed in the incident magnetic field.

Now, if the magnetic flux is changing with respect to time then:

$$
\frac{\Phi}{t}=\frac{B * A}{t}(4)
$$

This would permit the magnetic field to be changing in time. Furthermore, if the intensity of the magnetic field is subject to change, through amplitude modulation, then Equation 4 would take on the form of Maxwell-Faraday equation for induction. That is to say that an amplitude modulated, time varying electromagnetic field would elicit a phase change in a particle's wave-function through Faraday Induction and would be represented in the Arahanov-Bohm-Faraday equation:

$$
\frac{\Delta \varphi}{t}=\frac{q B A}{\hbar t}(5)
$$

The derived equation for Arahanov-Bohm induced phase change is remarkably similar to the equation relating phase change in coherent domain water, outlined by Del Guidice et al. [19], where an applied electromagnetic field generates an electric potential as described by the equation:

$$
\mathrm{V}=\frac{\hbar}{2 \pi e} \frac{d \phi}{d t}(6)
$$

Where $\frac{d \phi}{d t}$ is the changing magnetic flux, $\hbar$ is reduced Planck's constant, and $e$ is the elementary unit charge.

This implies that water, or at least a coherent state of water, in the presence of an external electromagnetic field, can have its phase modulated thus allowing for the potential of information storage or processing.

It should be noted that the original value for phase change $(\Delta \varphi)$ is a dimensionless value. However, if the phase changes with respect to time, then its dimensions become $1 / \mathrm{s}$, or the equivalent of the 'frequency of phase change'. Alternatively, the resulting dimensions could be interpreted as the number of phase transitions evolving in time (process). The time-varying change of phase will be herein referred to, interchangeably, as the "dynamic phase" or the "time-varying change of phase". 


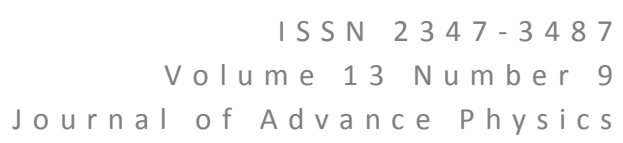

Evaluating Equation 5 suggests that, changing the intensity (degree of amplitude modulation) of the applied EMF would result in an increase in magnitude of the 'dynamic phase' resulting in an alteration of potential degrees of freedom. Additionally, changing the point duration (e.g., duration of current presentation, timing of the field) of the applied field would also result in a constriction (when the point duration is increased) or dilation (when the point duration is decreased) of the 'dynamic phase' resulting in a change of the available degrees of freedom. Finally, changes in the structure, and incidentally the physical geometry, of the matter under observation which is penetrated by the incident magnetic field, will result in a change in the 'dynamic phase'. Taken together, this system describes the "application geometry" of the applied EMF by accounting for intensity, timing, and geometric structure of the field where the resultant synthesis of these parameters are manifested into a pattern of time-varying phase modulations.

Phase modulation is the process by which the phase of a carrier wave is altered to follow the changing amplitude of an incident signal. The peak amplitude and frequency of the carrier wave remains constant, but as the amplitude of the information signal changes the phase of the carrier wave changes correspondingly. If this were applied to a physical apparatus capable of undergoing a change in electric potential, we would call it Faraday induction. In this instance, we would induce a change in one wave through the application of another. This convergence provides the necessary parameters to potentially modulate and encode information in an incident signal in order to appreciably change the structure-function relationship of the observed system. Alternatively, any system which demonstrates the capacity to generate its own magnetic potential, via the production of its own magnetic field, has the ability to self-modulate producing a complex, time-varying dynamic phase. The systematic evaluation of pertinent, biologically relevant systems may be revealing.

Hydrogen is the most abundant element, and consequently matter, pervading the Universe. In fact, Persinger [41] demonstrated that the ratio of the mass of the Universe $\left(10^{52} \mathrm{~kg}\right)$ to its volume $\left(10^{78} \mathrm{~m}^{3}\right)$ results in a density of approximately $10^{-26} \mathrm{~kg} \cdot \mathrm{m}^{-3}$, or the equivalent of roughly 1 proton or hydrogen atom per unit volume. Perhaps the dynamic phase of hydrogen is revealing of the Nature of the set or reflects some emergent property which can be appreciated in a local space-time reference. Here we assume that any given hydrogen atom, in the Universe, is immersed in the intergalactic magnetic field whose intensity ranges from $10^{-12}$ to $10^{-15} \mathrm{~T}$. If we assume that an intergalactic magnetic field penetrates a given area that is equivalent to the cross-sectional area of the neutral hydrogen line $(21.12 \mathrm{~cm})$ and whose temporal parameter is also the neutral hydrogen line $\left(1.42 \mathrm{GHz}\right.$ or $\left.7.04 \cdot 10^{-10} \mathrm{~s}\right)$ and we substitute these values in equation 5 , the resultant dynamic phase would be in the order of $0.965 \cdot 10^{11} \mathrm{~Hz}$. If we consider the dynamic phase to be akin to a frequency, which is really just the number of iterations of ' $X$ ' events per unit time, then we would be able to compute an energy and wavelength associated with the hydrogen dynamic phase. Using the Planck-Einstein equation (equation 7 ) provided that the frequency is $0.965 \cdot 10^{11} \mathrm{~Hz}$ the resultant energy would be in the order of $6.39 \cdot 10^{-23} \mathrm{~J}$, with a corresponding wavelength of $3.1 \cdot 10^{-3} \mathrm{~m}$. The former is approximately twice the value of Landauer's limit for a temperature of $4 \mathrm{~K}$ (cosmic microwave background; $3.80 \cdot 10^{-23} \mathrm{~J}$ ). In addition, bio-electromagnetic fields falling within the millimeter range were postulated by Devyatkov et al., [35] and Betskii et al., [4] to have been generated by the geometric and mechanical properties and asymmetries of polar membranes. Furthermore, Fröhlich [27] theorized that electromagnetic field coupling within the millimeter range would recruit resonant, vibrational processes of biomolecular structures in response to the application of applied electromagnetic fields.

In addition, the resulting dynamic phase, that is in the order of $10^{11} \mathrm{~Hz}$, would fall within the range of proton movement and hydronium complex formation as denoted by Pollack [46, 47, 48] and Del Guidice [22, 33] as well as others $[17,23]$.

$$
E=h \cdot v=h \cdot c / \lambda(7)
$$

Where $\mathrm{h}$ is Planck's constant, $\mathrm{v}$ is frequency $(\mathrm{Hz}), \mathrm{c}$ is the speed of light, and $\lambda$ is the wavelength.

Next, we consider the electron and assume that the energy associated with its rotation around a Bohr nucleus can be approximated using the equation of kinetic energy as function of rotation around a circle.

$$
E=m \cdot 2 \cdot \pi \cdot r^{2} \cdot f^{2}(8)
$$

Where $\mathrm{E}$ is energy, $\mathrm{m}$ is mass, $\mathrm{r}$ is the Bohr radius, and $f$ is the frequency of rotation.

For a mass of $9.11 \cdot 10^{-31} \mathrm{~kg}$, a radius of rotation equal to $52.9 \cdot 10^{-12} \mathrm{~m}$, and frequency of equivalent of $1.52 \cdot 10^{-16} \mathrm{~s}$, the resultant energy would in the order of $6.93 \cdot 10^{-19} \mathrm{~J}$, well within the range of visible light. The internal magnetic field generated by an electron as it orbits the central proton, can be approximated by the quotient of the rotational energy and its orbital magnetic moment $\left(9.27 \cdot 10^{-24} \mathrm{~A} \cdot \mathrm{m}^{2}\right)$, generating a field whose strength would be $7.48 \cdot 10^{4} \mathrm{~T}$. Again, using our dynamic phase equation (Equation 5), and substituting values of $7.48 \cdot 10^{4} \mathrm{~T}$ for $\mathrm{B}$, whose area is defined by the Bohr radius $\left(3.51 \cdot 10^{-20} \mathrm{~m}^{2}\right)$, and the rotation time (field pattern) of $1.52 \cdot 10^{-16} \mathrm{~s}$, results in a dynamic phase of $2.63 \cdot 10^{16} \mathrm{~Hz}$, the time equivalent to $3.79 \cdot 10^{-17} \mathrm{~s}$, or within the order of half the rotation time around the atom. Persinger [43], postulated that the electron in orbit around the Bohr atom spent half of its rotation in the particle (matter) state, while for the other half of the time the electron took on the state of its wave-function. In essence, this would suggest that the electron is self 


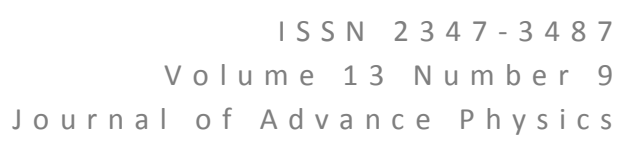

modulating with dynamic phase transitions occurring throughout half its rotation. The most ideal candidate that could allow for dynamic phase shifts would be the waveform equivalent of the electron. Again assuming that this phase transition may involve, or be mediate by photons, one can calculate the wavelength and energy equivalent of $2.63 \cdot 10^{16} \mathrm{~Hz}$ using Equation 7. The energy carried by a $2.63 \cdot 10^{16} \mathrm{~Hz}$ photon would be $1.74 \cdot 10^{-17} \mathrm{~J}$, with a corresponding wavelength of 1.14 $.10^{-8} \mathrm{~m}$. This latter wavelength is within the average thickness of the cell membrane and Exclusion Zones of water [47] Incidentally, this value corresponds to the peak wavelength shift of water exposed to a physiologically patterned weak EMF in the dark for 28 days as measured by fluorescence microscopy [37].

In terms of biological relevance, if a cell has a primary operational energy of $2.0 \cdot 10^{-20} \mathrm{~J}$ [39], and whose size is approximately $10^{-5} \mathrm{~m}$, we can calculate the magnetic field component by looking at Equation 9 and solving for $\mathrm{B}$.

$$
\mathrm{E}=\frac{B^{2}}{2 \mu} \cdot \mathrm{V}(9)
$$

Where $E$ is the energy, $B$ is the intensity of the magnetic field, $u$ is the permeability of free space, and $V$ is the volume.

The intensity derived from solving for $\mathrm{B}$, would be $6.93 \cdot 10^{-6} \mathrm{~T}$. Provided that the fundamental frequency of operation of a cell is approximately $10 \mathrm{~Hz}$ then, solving for Equation 5, the resulting dynamic phase would yield a value of $33 \mathrm{~Hz}$. A $33 \mathrm{~Hz}$ dynamic phase approaches the $20-25 \mathrm{msec}$ refresh rate of consciousness [54] and can be elicited in samples of spring water exposed to microTesla intensity, physiologically patterned electromagnetic fields [36]. This would imply that consciousness may be the dynamic phase of a single cell's primary operational frequency. Furthermore, this would suggest that the whole is reflected in the sum of its parts $(\Sigma n=n)$ [42] and is manifested as that the holographic representation of information within a subset of lower levels of discourse. Burke [8] discussed that the record of interference patterns represented in a holographic state, contains a unique description of the parameters of phase, direction, and polarity of the electromagnetic radiation which was used in order to generate the holographic equivalent of a given structure. That is to say, that the information of that given system can be represented and stored within the holographic equivalent of the structure under investigation. In this vane, we consider electromagnetic radiation, in any form, as a possible mechanism by which a hologram can be manifested.

The contribution of dynamic phase to the storage and representation of information may involve redundancies or aggregates of units in order to be effectively represented. In fact, in order to accommodate a dynamic phase of $10^{12}$ (hydrogen line) or even $10^{16}$ (electron circling the Bohr atom), would require approximately $10^{11}$ to $10^{14}$ cells that were "phase locked". Additionally, if one changed the intensity of the incident field to that of $3.0 \cdot 10^{-5} \mathrm{~T}$, with a point duration of $10^{-3} \mathrm{~s}\left(1\right.$ or $3 \mathrm{msec}$ ) the dynamic phases allowed would be $10^{4} / \mathrm{cell}$ and would require approximately $10^{8}$ to $10^{12} \mathrm{cells}$ in order to accommodate. The former value falls within the number of cells calculated to be involved with consciousness [53], and the latter reflects the total number of cells within the cerebral manifold.

\section{THE INTERACTION OF MAGNETIC ENERGY AND DYNAMIC PHASE}

Thus far we have demonstrated the relationship between an incident, or applied, EMF as a means to manipulate or change the phase of a particle's wave-function. However, we can also relate a change in phase, and consequently a change in dynamic phase, as a function of energy. If we re-arrange Equation 9 to solve for the intensity of the magnetic field (Equation 10) and substitute this relationship into Equation 5, the result corresponds to a change in dynamic phase contingent upon the energy contained within the magnetic field.

$$
\mathrm{B}=\sqrt[2]{\left(\frac{E * 2 \mu}{m^{3}}\right)}(10)
$$

Where $E$ is the energy, $B$ is the intensity of the magnetic field, $u$ is the permeability of free space, and $V$ is the volume.

$$
\frac{\Delta \varphi}{t}=\frac{q A \sqrt[2]{\left(\frac{E * 2 \mu}{m^{3}}\right)}}{\hbar t}(11) .
$$

And if were to isolate for the energy, which would then be derived from the area penetrated by the magnetic field, spatial structure of the observed unit, the volume in which the magnetic field is generated, the point duration (frequency) of the field, and the dynamic phase (Equation 12).

$$
\mathrm{E}=\frac{\left(\frac{\varphi \mathrm{h} t}{q A}\right)^{2} * m^{3}}{2 \mu}(12)
$$




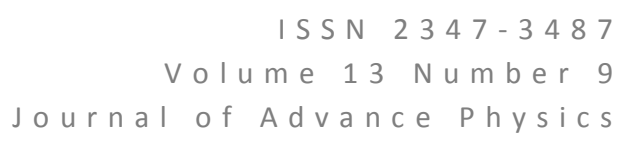

If we substitute the values of $0.965 \cdot 10^{11} \mathrm{~Hz}$ for dynamic phase, an area equivalent to the cross-section of the hydrogen line wavelength $(21.12 \mathrm{~cm})$, the volume equivalent of the neutral Hydrogen line, and the time equivalent of $1.42 \cdot 10^{9} \mathrm{~Hz}\left(7.04 \cdot 10^{-10}\right.$ seconds), the resultant energy would be in the order of $7.39 \cdot 10^{-21} \mathrm{~J}$. This latter value is within the range of energy necessary to transform (gain or lose) 1 bit of information according to Landauer's limit for a system operating at $37^{\circ} \mathrm{C}$. Posit, if you will, the potential overlap between the neutral Hydrogen and the operational parameters of the human cerebrum. This would provide a means in which one could "store" information in a non-local means. Furthermore, a magnetic field whose energy is $10^{-20} \mathrm{~J}$, applied over the cerebral geometry (volume and area) with a timing in the order of 1 or $3 \mathrm{msec}$ would produce a dynamic phase in the order of $\sim 10^{11}$, or that which was derived from the hydrogen line. The resulting intensity of the applied EMF would be in the order of $10^{-5} \mathrm{~T}$, or those which have been employed by our laboratory.

\section{CASMIR-MAGNETIC PRESSURE AND PHASE CHANGE IN TIME}

We have demonstrated that, in effect, we can modulate the dynamic phase of a system with the application of exogenous EMFs, and we have demonstrated the potential of internal (self-generated) fields with the possibility to effectively modulate the phase, and thus the information, within a given space-time system. Here we examine the generation of phase modulation through quantum phenomena.

When examining a set of non-conducting surfaces, whose separation is much smaller than the surface area of the conductor, a discrete pressure is generated and can be modeled using the Equation 13.

$$
\mathrm{F} / \mathrm{A}=\frac{-h c \pi^{2}}{240 a^{4}}(13)
$$

Where $a$ is the separation between the plates, $\hbar$ is reduced Planck's constant, and $\mathrm{c}$ is the speed of light.

In order to consider the influence of the pressure generated by a Casimir effect [7] to affect the phase, and ultimately the dynamic phase, of a particle then we must consider this pressure to be related to a magnetic field. Then the magnetic pressure of a system is calculated by (Equation 14):

$$
F / A=B^{2} / 2 \mu(14)
$$

Substituting Equation 14 into Equation 13 gives us:

$$
\frac{\mathrm{B}^{2}}{2 \mu}=\frac{-h c \pi^{2}}{240 a^{4}}(15)
$$

If we consider that the intensity of the applied EMF produces a given dynamic phase change, provided in our calculations above, then this might provide a necessary insight into the separation of given structures (non-conducting plates) that would accommodate such a dynamic phase change. For instance, if one assumes a magnetic field strength of $10^{-5} \mathrm{~T}$, the resulting separation between cells, the surface which was necessary in order to produce this intensity, would be in the order of $\sim 1 \mathrm{um}$. This would be within the spatial extent of Pollack's exclusion zones [10, 12], as well as within the Bohr's spatial limit for thinking and consciousness [43]. Additionally, if you were to take the intensity of the magnetic field generated by the electron rotating around a Bohr atom, with an intensity of $\sim 10^{5} \mathrm{~T}$, the resultant separation would be in the order of $10^{-11} \mathrm{~m}$, or with the spatial extent of the Bohr radius.

If we postulate that our dynamic or time-varying phase change is related to the translation of information through means of phase-modulation then, the parameters by which a change in phase can occur is on a wave. In this light, we can relate our $10^{16} \mathrm{~Hz}$ time-varying phase change to processes ongoing at the level of packets of photons. Here then we can calculate the relative distance between successive phase shifting points. The wavelength of light that would be associated with a dynamic phase of $10^{16} \mathrm{~Hz}$ would be $10^{-8} \mathrm{~m}$. This value may correspond to a spatial distance necessary to observe the discrete shifts in phase. That is to say, that the spatial equivalence of $10^{-8} \mathrm{~m}$ may be necessary in order to interpret the information stored within a time-varying phase change of $10^{-16} \mathrm{~Hz}$.

\section{THE NECESSARY PARAMETERS TO INTERPRET INFORMATION WITHIN A PHASE}

We have demonstrated that the wavelength of light equivalent for a dynamic phase of $10^{16} \mathrm{~Hz}$ is $10^{-8} \mathrm{~m}$. Here we make the argument that cell membranes have an oscillatory component which accommodates a magnetic moment. Dotta et al., [24], calculated that the intrinsic magnetic moment of a cell would range between $10^{-23}-10^{-24} \mathrm{~A} \cdot \mathrm{m}^{2}$. The application of a $10^{16} \mathrm{~Hz}$ phase shift equivalent energy would be in the order of $10^{-18}-10^{-17} \mathrm{~J}$. When the quotient of the energy of phase change, and cell magnetic moment is equated the resultant electromagnetic field intensity would range between 10 ${ }^{6}-10^{-5} \mathrm{~T}$. These latter electromagnetic intensities are within the range necessary to elicit changes in peak fluorescence readings in spring water exposed to patterned electromagnetic fields in the dark [37]. Furthermore, data suggests that the 


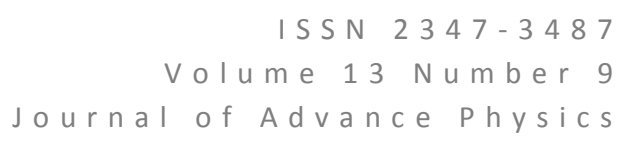

appropriate combination of patterned electromagnetic fields and light applied to B16-BL6 cells, increased photon emissions in these cells and were found to be highly correlated the incident field/light energy [31] reflecting a potential ability for cells to store electromagnetic radiation, a phenomenon originally postulated by Popp [50]. This may implicate the cell, or at least the cell's constituent structures, as a potential translator of information stored within a phase-modulated pattern.

Considering Del Guidice's interpretation of phase change, generated through electric potential energy, as being modulated by the formation of coherent domains in water [18, 19], it could be argued that water, in a coherent state, has the capacity to interact with the process underlying the transformation of electrical potential to phase change. Experimentally, coherent domain water was expressed to have the capacity to undergo a change in phase with respect to incident electromagnetic potential $[20,21]$. Provided that the cell membrane exhibits the spatial structure necessary to process the dynamic, time-varying phase of a magnetic field and water, in a coherent state, can contribute to integration of information contained within a dynamic phase, we postulate that the dynamics of the membrane-water interface maybe the receiver/transformer necessary to decode changes in phase.

Furthermore, if one considers light to be involved in this process and we have demonstrated that a space of $10^{-8}$ $\mathrm{m}$ as being potentially sufficient to interpret the information stored within the time-varying phase with a rate of $10^{16}$ events per unit time, then we suggest that the cell membrane or a layer of water approximately 10 units thick, would set the parameters for separation of information within a phase. The time it takes light to travel a distance of $10^{-8} \mathrm{~m}$ is approximately $10^{-16} \mathrm{~s}$. The relationship between this and the Bohr-orbital rotational time was detailed by Persinger and Lafrenie [45]. This would potentially allow the time-varying phase to be read, or interact with, processes occurring at the level of the Bohr magneton. Alternatively, the coherent, initially phase-locked, activity of $10^{4}$ water molecules, whose limit of information processing is determined by association and dissociation of the hydronium complex, or $10^{12} \mathrm{~Hz}$ would correspond to a mass of approximately $10^{-22} \mathrm{~kg}$. The volume, provided that the density of water is $10^{3} \mathrm{~kg} \cdot \mathrm{m}^{-3}$, would be in the order of $10^{-25} \mathrm{~m}^{3}$, the cubed root of which would correspond to a linear distance of approximately $10^{-8} \mathrm{~m}$. This may suggest that nano-clusters of water [51], or even smaller segments, along the membrane may be used in order to accommodate higher information processing.

Finally, when looking at the Casimir effect, which allows for the creation of real particles from virtual particles from zero-point fluctuations, (Equation 16), and re-arranging to solve for the distance of separation between cells, whose diameter is $10 \mathrm{um}$, provided the resultant energy is $10^{-17}$ to $10^{-18} \mathrm{~J}$ yields a value in the order of $1.42 \cdot 10^{-6}$ to $3.14 \cdot 10^{-6} \mathrm{~m}$. These values fall within the range Bohr's postulate of the quantum of energy for consciousness and thinking [43] as well as in the order of error with respect to the peak frequency most associated with effective inflation of the exclusion zone of water around a boundary $[10,11]$. In addition, this separation is typically what is found between synaptic connections in the neural network associated with memory (Crosby, 1962).

$$
E=\frac{-h c \pi^{2}}{720 a^{3}} \mathrm{~A}(16)
$$

Where $\mathrm{E}$ is the energy created by zero point fluctuations, $\mathrm{a}$ is the distance between plates (cells; $\mathrm{m}$ ) and $\mathrm{A}$ is the surface area of the plates (cells; $\mathrm{m}^{2}$ ).

\section{CONCLUSION}

We have demonstrated the potential of an amplitude-modulated signal can produce a time-varying, changing electromagnetic potential. This interaction of electromagnetic potential can induce a change in phase of a charged particle in time. Thus resulting in a rate of change in phase, or tame-varying, dynamic phase. In addition, we have suggested that information can be stored within these time-varying phase modulations. The primary carrier associated with this information's transmission would be the photon.

The Casimir phenomenon generated from the presence of a magnetic pressure of $10^{-5} \mathrm{~T}$, would be accommodated by a separation between plates in the order of $1 \mathrm{um}$. When accommodating for the energy equivalent of the time-varying phase of the Bohr magneton, $2.62 \cdot 10^{16} \mathrm{~Hz}$, the value of the separation between Casimir boundaries, provided the surface area is approximately that of the cell, would range between 1.42 to 3.14 micrometers. Furthermore, we have presented convergent quantification that may also implicate the cell membrane as a functional filter of photontransmitted, phase modulated information. Water along a boundary, provided that it is devoid of mechanical stimulation, may also suffice as an information receiver.

\section{ACKNOWLEDGEMENTS}

The author would like to thank Dr. Michael Persinger for discussions and interpretative contributions to the above document. 


\section{REFERENCES}

1. Aharonov, Y., \& Bohm, D. (1959). Significance of electromagnetic potentials in the quantum theory. Physical Review, 115(3), 485

2. Apolonski, A., Poppe, A., Tempea, G., Spielmann, C., Udem, T., Holzwarth, R. H. T. W., ... \& Krausz, F. (2000). Controlling the phase evolution of few-cycle light pulses. Physical Review Letters, 85(4), 740.

3. Bassi, A., Lochan, K., Satin, S., Singh, T. P., \& Ulbricht, H. (2013). Models of wave-function collapse, underlying theories, and experimental tests. Reviews of Modern Physics, 85(2), 471.

4. Betskii, O.B., Golant, M.B., Devyatkov, N.D., (1988). Millimeter Waves in Biology, in Russian, Millimetrovye Volny V Biologii. No. 6 in Fizika. Znanie, Moskva.

5. Berger, G., Dietz, M., \& Denz, C. (2008). Overloaded phase-code multiplexing for volume holographic storage. Optics letters, 33(11), 1252-1254.

6. Bohr, N. (1918). On the quantum theory of line spectra, part I: On the general theory. Сборник статей к мультимедийному электронному учебно-методическому комплексу по дисциплине «физика атома и атомных явлений»/отв. ред. Шундалов МБ; БГУ, Физический факультет.

7. Bordag, M., Mohideen, U., \& Mostepanenko, V. M. (2001). New developments in the Casimir effect. Physics reports, 353(1), 1-205.

8. Burke, H. E. (1986). Radiant Energy. In Handbook of Magnetic Phenomena (pp. 124-160). Springer Netherlands.

9. Cahn, C. (1959). Performance of digital phase-modulation communication systems. IRE Transactions on Communications Systems, 7(1), 3-6.

10. Chai, B., Yoo, H., \& Pollack, G. H. (2009). Effect of radiant energy on near-surface water. The Journal of Physical Chemistry B, 113(42), 13953-13958.

11. Chai, B.; Pollack, G.H. (2010). Solute-Free Interfacial Zones in Polar Liquids. J. Phys. Chem. B 114, 5371-5375.

12. Chai, B.; Zheng, J.; Zhao, Q.; Pollack, G.H. (2008). Spectroscopic studies of solutes in aqueous solution. J. Phys. Chem. A, 112, 2242-2247.

13. Cifra, M., Fields, J. Z., \& Farhadi, A. (2011). Electromagnetic cellular interactions. Progress in biophysics and molecular biology, 105(3), 223-246.

14. Chraplyvy, A. R., Tkach, R. W., Buhl, L. L., \& Alferness, R. C. (1986, February). Phase modulation to amplitude modulation conversion of CW laser light in optical fibers. In Optical Fiber Communication Conference (p. PD10). Optical Society of America.

15. Crosby, E. C. (1962). Correlative anatomy of the nervous system. Macmillan.

16. De Broglie, L. (1923). Waves and quanta. Nature, 112(2815), 540-540.

17. Decoursey, T. E. (2003). Voltage-gated proton channels and other proton transfer pathways. Physiological reviews, 83(2), 475-579.

18. Del Giudice, E., \& Preparata, G. (1995). Coherent dynamics in water as a possible explanation of biological membranes formation. Journal of Biological Physics, 20(1-4), 105-116.

19. Del Giudice, E. D., Spinetti, P. R., \& Tedeschi, A. (2010). Water dynamics at the root of metamorphosis in living organisms. Water, 2(3), 566-586.

20. Del Giudice, E., De Ninno, A., Fleischmann, M., Mengoli, G., Milani, M., Talpo, G., \& Vitiello, G. (2005). Coherent quantum electrodynamics in living matter. Electromagnetic Biology and Medicine, 24(3), 199-210.

21. Del Giudice, E., Tedeschi, A., Vitiello, G., \& Voeikov, V. (2013). Coherent structures in liquid water close to hydrophilic surfaces. In Journal of Physics: Conference Series (Vol. 442, No. 1, p. 012028). IOP Publishing.

22. Del Giudice, E., Stefanini, P., Tedeschi, A., \& Vitiello, G. (2011). The interplay of biomolecules and water at the origin of the active behavior of living organisms. In Journal of Physics: Conference Series (Vol. 329, No. 1, p. 012001). IOP Publishing.

23. DeMeo, J. (2011). Water as a Resonant Medium for Unusual External Environmental Factors. Water, 3, 1-47.

24. Dotta, B. T., Lafrenie, R. M., Karbowski, L. M., \& Persinger, M. A. (2014). Photon emission from melanoma cells during brief stimulation by patterned magnetic fields: is the source coupled to rotational diffusion within the membrane. General Physiology and Biophysics, 33, 63-73.

25. Einstein, A., \& Infeld, L. (1949). On the motion of particles in general relativity theory.

26. Fang, K., yu, Z., and Fan, S. (2012). Photonic Arahanov-Bohm effect based on dynamic modualtion. Phys. Rev. Lett. 108, 153901. 
27. Fröhlich, H., 1980. The biological effects of microwaves and related questions. Advances in Electronics and Electron Physics 53, 85e152.

28. Heisenberg, W., Bohr, N., \& Rundschau, N. (1958). Copenhagen Interpretation. Physics and philosophy.

29. Ikonen, E., Helisto, P., Hietaniemi, J., \& Katila, T. (1988). Magnetic phase modulation of recoilless gamma radiation by nuclear Zeeman effect. Physical review letters, 60(7), 643.

30. Janson, L., Fithian, W., \& Hastie, T. J. (2015). Effective degrees of freedom: a flawed metaphor. Biometrika, 102(2), 479-485. http://doi.org/10.1093/biomet/asv019

31. Karbowski, L. M., Murugan, N. J., \& Persinger, M. A. (2016). Experimental Evidence That Specific Photon Energies Are" Stored" in Malignant Cells for an Hour: The Synergism of Weak Magnetic Field-LED Wavelength Pulses. Biology and Medicine, 8(1), 1.

32. Lowe, D. (1998). Characterising complexity by the degrees of freedom in a radial basis function network. Neurocomputing, 19(1), 199-209.

33. Marchettini, N., Del Giudice, E., Voeikov, V., \& Tiezzi, E. (2010). Water: A medium where dissipative structures are produced by a coherent dynamics. Journal of theoretical biology, 265(4), 511-516.

34. Mérolla, J. M., Mazurenko, Y., Goedgebuer, J. P., Porte, H., \& Rhodes, W. T. (1999). Phase-modulation transmission system for quantum cryptography. Optics letters, 24(2), 104-106.

35. Millimetrovye volny i ikh rol v processakh zhiznedeyatelnosti. In: Devyatkov, N.D., Golant, M.B., Betskii, O.B. (Eds.), Millimeter Waves and Their Role in Life Processes. Radio i svyazi, Moskva in Russian.

36. Murugan, N. J., Karbowski, L. M., \& Persinger, M. A. (2014). Serial pH increments ( 20 to 40 milliseconds) in water during exposures to weak, physiologically patterned magnetic fields: implications for consciousness. Water, $6,45-60$.

37. Murugan, N. J., Karbowski, L. M., Lafrenie, R. M., \& Persinger, M. A. (2015). Maintained Exposure to Spring Water but not Double Distilled Water in Darkness and Thixotropic Conditions to Weak $(\sim 1 \mu \mathrm{T})$ Temporally Patterned Magnetic Fields Shift Photon Spectroscopic Wavelengths: Effects of Different Shielding Materials. Journal of Biophysical Chemistry, 6(1), 14-28.

38. Papapetrou, A. (1948, January). Einstein's theory of gravitation and flat space. In Proceedings of the Royal Irish Academy. Section A: Mathematical and Physical Sciences (Vol. 52, pp. 11-23). Royal Irish Academy.

39. Persinger, M. A. (2010). 10-20 Joules as a neuromolecular quantum in medicinal chemistry: an alternative approach to myriad molecular pathways?. Current Medicinal Chemistry, 17(27), 3094-3098.

40. Persinger, M. A. (2012a). Solutions for real values in Minkowski four-dimensional space may link macro-and micro-quantum processes in the brain. Neuroscience \& Biobehavioral Reviews, 36(10), 2334-2338.

41. Persinger, M.A., (2012b). Potential origins of a quantitative equivalence between gravity and light. Open Astron $\mathrm{J}$ 5:41-43.

42. Persinger, M. (2012c). The (Sum of $n$ )=n Concept And the Quantitative Support for the Cerebral-Holographic and Electromagnetic Configuration of Consciousness. Journal of Consciousness Studies, 19(11-12), 128-153.

43. Persinger, M. A. (2015). Thixotropic phenomena in water: quantitative indicators of Casimir-magnetic transformations from vacuum oscillations (virtual particles). Entropy, 17(9), 6200-6212.

44. Persinger, M. A., \& St-Pierre, L. S. (2015). The physical bases to consciousness: Implications of convergent quantifications. J. Sys. Integrat. Neurosci, 1, 55-64.

45. Persinger, M. A. and Lafrenie, R. M. 2014. The cancer cell membrane potentials as energetic equivalents to astrophysical parameters. Int. Lett. Chem. Phys. Astron. 17(1), 67-77.

46. Pollack, G.H.; Clegg, J. Unexpected linkage between unstirred layers, exclusion zones, and water. In Phase Transitions in Cell Biology; Pollack, G.H., Chin, W.C., Eds.; Springer Science \& Business Media: Berlin, Germany, 2008; pp. 143-152.

47. Pollack, G. H., Figueroa, X., \& Zhao, Q. (2009). Molecules, water, and radiant energy: new clues for the origin of life. International Journal of Molecular Sciences, 10(4), 1419-1429.

48. Pollack, G.H. (2010) Water, energy and life: Fresh views from the water's edge. Int. J. Des. Nat. Ecodyn., 5, 2729.

49. Pollet, T., Van Bladel, M., \& Moeneclaey, M. (1995). BER sensitivity of OFDM systems to carrier frequency offset and Wiener phase noise. IEEE Transactions on communications, 43(234), 191-193.

50. Popp, F. A. (1979). Coherent Photon Storage of Biological Systems. In F. A. Popp, H. Koenig and W. Prechka (Eds.), Electromagnetic Bio-Information (pp. 123-149). Munich-Wein-Baltimore: Urban and Schwarzenberg. 
51. Rasaiah, J. C., Garde, S., \& Hummer, G. (2008). Water in nonpolar confinement: from nanotubes to proteins and beyond. Annu. Rev. Phys. Chem., 59, 713-740.

52. Reeves, W. T. (1983). Particle systems-a technique for modeling a class of fuzzy objects. ACM Transactions on Graphics (TOG), 2(2), 91-108.

53. Rouleau, N., \& Dotta, B. T. (2014). Electromagnetic fields as structure-function zeitgebers in biological systems: environmental orchestrations of morphogenesis and consciousness. Frontiers in integrative neuroscience, 8.

54. Saroka KS, Persinger MA (2013) Potential production of Hughling Jackson's "parasitic consciousness" by physiologically-patterned weak transcerebral magnetic fields: QEEG and source localization. Epilep. Behav. 28: 395-407.

55. P.C. Sun, Y. Mazurenko, and Y, Fainman, Opt. Lett. 20, 1062 (1995)

56. Tegmark, M. (1993). Apparent wave function collapse caused by scattering. Foundations of Physics Letters, 6(6), $571-590$.

57. Tonomura, A., Osakabe, N., Matsuda, T., Kawasaki, T., Endo, J., Yano, S., \& Yamada, H. (1986). Evidence for Aharonov-Bohm effect with magnetic field completely shielded from electron wave. Physical Review Letters, 56(8), 792 